\title{
Peripherally inserted central venous catheter in upper extremities leads to an increase in D-dimer and deep vein thrombosis in lower extremities
}

Wanli Liu ${ }^{1,2}$, Lianxiang He ${ }^{1,2}$, Wenjing Zeng ${ }^{2,3}$, Liqing Yue ${ }^{1,2}$, Jie Wei ${ }^{2,3}$, Shuangshuang Zeng ${ }^{2,3}$, Xiang Wang ${ }^{2,3}$ and Zhicheng Gong ${ }^{2,3^{*}}$

\begin{abstract}
Background: The purpose of this study is to elucidate the association between peripherally inserted central venous catheter (PICC) in upper extremities and lower extremity deep venous thrombosis (LEDVT) by observing the changes in D-dimer.

Methods: This was a retrospective cohort study with 3452 patients (104 inserted with PICCs and 3348 without PICC) enrolled at the neurology department from April 1, 2017 to April 1, 2020. The patients underwent color Doppler ultrasound (CDU) and D-dimer examinations. LEDVT-related factors and D-dimer value were analyzed before and after PICC insertion. The predictive value of D-dimer for LEDVT was also evaluated.

Results: Univariate logistic regression analysis showed that PICC insertion increased the risk of LEDVT by 9 times and promoted the increase of D-dimer by 5 times. After risk adjustment, multivariate logistic regression analysis showed that PICC insertion increased the risk of LEDVT by 4 times and tripled the risk of D-dimer increase. The concentration of D-dimer was significantly increased after PICC insertion. D-dimer was unsuitable for excluding venous thrombosis in patients inserted with PICCs.
\end{abstract}

Conclusions: PICC insertion increases the level of D-dimer and the risk of LEDVT. The risks of venous thrombosis need to be assessed in patients inserted with PICCs to ensure the expected clinical outcomes.

Keywords: Peripherally inserted central venous catheter, Lower extremity deep venous thrombosis, D-dimer, Neurology department

\footnotetext{
* Correspondence: gongzhicheng@csu.edu.cn

${ }^{2}$ Institute for Rational and Safe Medication Practices, National Clinical Research Center for Geriatric Disorders, Xiangya Hospital, Central South University, Changsha, Hunan, People's Republic of China

${ }^{3}$ Department of Pharmacy, Xiangya Hospital, National Clinical Research

Center for Geriatric Disorders, Central South University, Hunan 410008

Changsha, People's Republic of China

Full list of author information is available at the end of the article
}

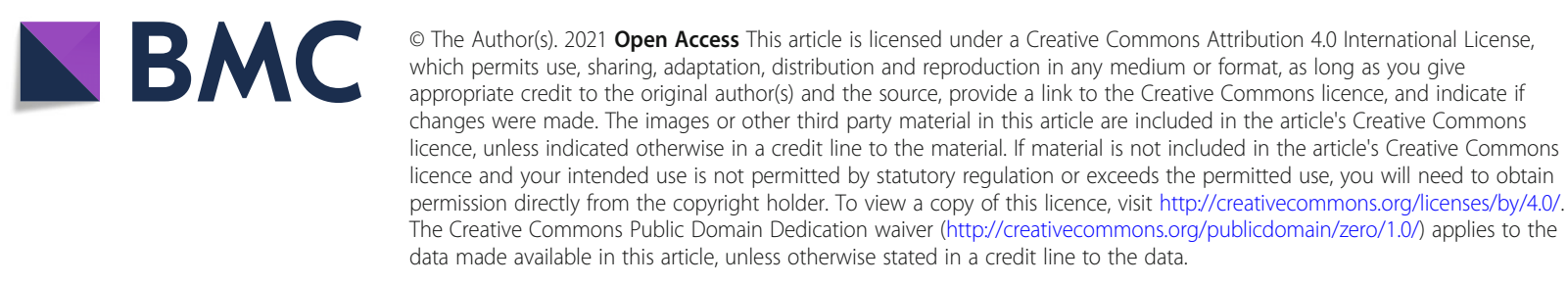




\section{Introduction}

A peripherally inserted central venous catheter (PICC) is a tube that extends to the superior vena cava or inferior vena cava through superior limb basilic vein, median cubital vein, cephalic vein, brachial vein or external jugular vein. A PICC can enter the body of a newborn at great saphenous vein of lower limb, temporal vein of head or retroauricular vein. The use of PICC can reduce the pain of repeated puncture and the incidence of drug extravasation, which is welcomed by clinical medical staff, patients and family members [1]. However, PICC insertion and displacement may damage the vascular intima [2]. PICC insertion-induced endothelial injury and stasis of blood flow coupled with medication-induced hypercoagulability constitute the Virchow's triad for thrombosis [3]. Deep venous thrombosis (DVT) is a venous thromboembolic disorder defined as "the formation of a blood clot within a deep vein" [4]. The incidence of PICC-related symptomatic upper extremity DVT (UEDVT) varies between 6 and $18 \%$, but it can reach $25 \%$ in a few circumstances such as malignancies $[5,6]$. The prevalence of asymptomatic thrombosis ranges from 35 to $71.9 \%$ [7-11].

PICC-related venous thrombosis is mainly mural thrombus and limited to the venous route where the catheter is located [12-14]. Increasing evidence has suggested that PICC insertion also increases the incidence of lower extremity DVT (LEDVT) and that PICC-related venous thrombosis can even exceed the range of infusion route $[2,3,15,16]$. However, there is no explanation for this clinical phenomenon.

D-dimer is a specific degradation product of cross-linked fibrin. An increase in D-dimer reflects the enhancement of coagulation and fibrinolysis system, which can be used as a sensitive indicator of hypercoagulability $[12,17]$. D-dimer is augmented in catheter-related venous thrombosis [18, 19], and the normal value of D-dimer can be used to exclude venous thrombosis $[18,20-23]$. Nonetheless, it is not clear whether D-dimer is the medium or by-product of catheterrelated venous thrombosis [15]. In this study, the association between PICC in upper extremities and LEDVT was investigated by observing the changes in D-dimer.

\section{Materials and methods}

\section{Patients and study design}

The study was approved by the ethics committee of Xiangya Hospital of Central South University $(202,004,327)$ and conducted according to the Helsinki Declaration of Ethical Principles for Medical Research Involving Human Subjects.

This retrospective case cohort study involved 3452 patients who received color Doppler ultrasound (CDU) in the neurology department of Xiangya Hospital of Central South University, Hunan Province from April 1,
2017 to April 1, 2020. The clinical characteristics of the patients are listed in Table 1.

The inclusion criteria of research objects were as follows: aged $\geq 18$ years, admitted to the neurology department and receiving CDU from April 1, 2017 to April 1, 2020. The exclusion criteria were: (1) under 18 years old; (2) pregnant; (3) inserted with a PICC in the lower extremity; (4) diagnosed with venous thromboembolism within six months before admission. All the 3452 patients were indexed and given a Caprini score and routine D-dimer test at admission to the neurology department. Patients at high risk of venous thrombosis underwent $\mathrm{CDU}$ at admission. PICCs were used for long-term infusion in patients having poor vascular conditions such as unclear superficial veins of upper extremities and difficulty in peripheral short catheter insertion. Only 104 of the 3452 patients were inserted with PICCs.

CDU was performed at admission on unconscious patients or on those with a high-risk score or suspected symptoms of DVT such as swelling, pain, tenderness and pyrexia of lower extremities. During hospitalization, CDU was performed on patients when they showed thrombotic symptoms such as pain and swelling of lower extremities or on long-term bedridden patients. All the 3452 patients underwent CDU, among which 1360 patients received D-dimer test within seven days before the CDU examination. Only 101 of the 1360 patients were inserted with PICCs.

In summary, all patients received blood vessel assessment at admission/before infusion for deciding the use of PICC. PICC insertion was required when patients had poor vascular conditions such as unclear superficial

Table 1 Clinical characteristics of all patients

\begin{tabular}{lll}
\hline Factors & PICC & NO PICC \\
& $\boldsymbol{n}=104$ & $\boldsymbol{n}=3348$ \\
\hline Age (year, mean \pm standard deviation) & $62.0 \pm 14.0$ & $61.5 \pm 13.6$ \\
Male & $61(58.7 \%)$ & $2036(60.8 \%)$ \\
Malignant tumor & $7(6.7 \%)$ & $120(3.6 \%)$ \\
Recent surgery & $18(17.3 \%)$ & $222(6.6 \%)$ \\
Cerebral hemorrhage & $30(28.8 \%)$ & $206(6.2 \%)$ \\
Ischemic stroke & $32(30.8 \%)$ & $1581(47.2 \%)$ \\
Parkinson's disease & $2(1.9 \%)$ & $228(6.8 \%)$ \\
Infection & $6(5.8 \%)$ & $331(9.9 \%)$ \\
BMl $\geq 25$ & $9(8.6 \%)$ & $22(0.7 \%)$ \\
Unconsciousness & $80(76.9 \%)$ & $847(25.3 \%)$ \\
Critical illness & $11(10.6 \%)$ & $163(4.9 \%)$ \\
\hline
\end{tabular}

Note: PICC refers to peripherally inserted central venous catheter; $B M I$ refers to body mass index; malignant tumor refers to cases treated within the prior six months; recent surgery refers to neurosurgery (over two hours) performed within six months; critical illness refers to the circumstance where patients have a high risk of disease variation or death 
veins of upper extremities and difficulty in peripheral short catheter insertion. CDU was performed within $24 \mathrm{~h}$ on patients showing swelling, redness, pain, fever and other thrombotic symptoms during the catheterization. Comatose or bedridden patients without thrombotic symptoms underwent general ultrasound examination by nurses regularly once a week during the catheterization; CDU was further performed if the assessment result indicated suspected venous thrombosis.

\section{D-dimer test}

D-dimer test was performed within seven days after catheterization. Blood samples for D-dimer test were obtained before CDU examination. The blood was drawn from the antecubital vein with a clean 22-gauge butterfly needle to a $3-\mathrm{ml}$ plastic tube containing $0.3 \mathrm{ml}$ of $0.106 \mathrm{M}$ trisodium citrate. The tube was gently inverted to mix the blood with trisodium citrate for three to six times. The whole blood was centrifuged at $2000 \times \mathrm{g}$ for $20 \mathrm{~min}$ at $20{ }^{\circ} \mathrm{C}$. The automated and rapid STA Liatest ${ }^{\circ}$ D-dimer assay was used for measurement of D-dimer. The STA Liatest ${ }^{\circ}$ assay has high sensitivity (median value $\geq 95 \%$ ) which meets the requirements of the Food and Drug Administration and can be used to rule out venous thrombosis [24, 25]. The cut-off value for negative DVT was $0.5 \mathrm{mg} / \mathrm{L}$. The detection methods or reagents adopted by the blood laboratory of the hospital remained unchanged during the research period.

\section{CDU}

CDU was performed within seven days after D-dimer test by an experienced CDU technician using Philips Epiq 5 and a high-resolution linear array transducer (9-13 MHz). The criteria for the diagnosis of venous thrombosis were as follows [26-28]: the lumen cannot be compressed despite firm compression with the transducer probe; defective blood flow signal in the lumen; solid return in the lumen sound; disappearance or weakening of the spent response; phase change in the loss of blood spectrum; disappearance or weakening of the blood flow in the distal limb by squeezing.

\section{Statistical analysis}

Descriptive statistics for continuous variables and categorical variables were recorded as mean \pm standard deviation. Categorical variables were compared using Pearson's $X^{2}$ test. Continuous variables were compared using Student's $t$-test or Mann-Whitney rank sum test according to the normality of their distribution. Risk factors for LEDVT and D-dimer increase were studied by univariate and multivariate logistic regression analysis. A Student's $t$-test was used to compare the D-dimer value before and after PICC insertion. Risk assessments were presented as odds ratios (ORs) with $95 \%$ confidence intervals (CIs). The receiver operating characteristic (ROC) curve and the area under the curve (AUC) were used to evaluate the diagnostic capability and accuracy of D-dimer. All statistical analyses were conducted with SPSS (Version 18; SPSS, Central South University, Hunan, China). A $P$-value $<0.05$ (two-tailed) was considered significant.

\section{Result \\ PICC insertion increases the risk of LEDVT}

Among the 3452 patients, 270 patients (7.82\%, including 43 patients inserted with PICCs) were diagnosed with LEDVT by ultrasound. Among the 270 patients, 67 cases were diagnosed by CDU due to thrombotic symptoms during the short follow up, and 203 cases were asymptomatic LEDVTs diagnosed by vascular ultrasound. Among the 3182 patients (92.18\%) without LEDVT, 61 patients were inserted with PICCs. LEDVT occurred in 43 of 104 patients inserted with PICCs 6.8 days (an average value) after catheterization. The median time from index to LEDVT was five days. Univariate logistic regression analysis showed that malignancy, PICC insertion, recent surgery, body mass index $(\mathrm{BMI} \geq 25)$, unconsciousness, critical illness condition and diseases including ischemic stroke and Parkinson's disease were associated with the increased risk of LEDVT (Table 2). PICC insertion increased the risk of LEDVT by 9 times (Table 2, OR $=9.692$ [95\% CI: 6.414-14.646], $P=0.000)$. After adjustment of the risk factors, multivariate logistic regression analysis showed that PICC insertion increased the risk of LEDVT by 4 times (Table 2, OR = 4.268 [95 \% CI: 2.501-7.282], $P=0.000$ ).

\section{PICC insertion is associated with the increased risk of D-dimer} Patients with a high-risk Caprini score were inserted with PICCs. Among the 3452 patients, 1360 patients underwent D-dimer test within seven days after PICC insertion. Among the 1360 patients, 597 patients (43.90\%, including 80 patients inserted with PICCs) had a D-dimer value $>0.5 \mathrm{mg} / \mathrm{L}$, and 763 patients $(56.10 \%$, including 21 patients inserted with PICCs) had a Ddimer value $\leq 0.5 \mathrm{mg} / \mathrm{L}$. Univariate logistic regression analysis showed that age, male sex, malignancy, PICC insertion, recent surgery, unconsciousness and diseases including cerebral hemorrhage, ischemic stroke, critical illness condition, Parkinson's disease and infection were associated with the increased risk of D-dimer value (Table 3). PICC insertion promoted the increase of Ddimer value by 5 times (Table $3, \mathrm{OR}=5.467$ [95\% CI: 3.338-8.956], $P=0.000$ ). After adjustment of the risk factors, multivariate logistic regression analysis showed that PICC insertion promoted the increase of D-dimer value more than threefold (Table 3, OR $=3.354$ [95\% CI: 1.773-6.346], $P=0.000)$. 
Table 2 Logistic regression analysis of LEDVT-related factors

\begin{tabular}{|c|c|c|c|c|c|c|}
\hline \multirow[t]{2}{*}{ Factors } & \multirow{2}{*}{$\begin{array}{l}\text { LEDVT } \\
n=270\end{array}$} & \multirow{2}{*}{$\begin{array}{l}\text { NO LEDVT } \\
n=3182\end{array}$} & \multicolumn{2}{|l|}{ Univariate } & \multicolumn{2}{|l|}{ Multivariate } \\
\hline & & & OR $(95 \% \mathrm{Cl})$ & $P$ & OR $(95 \% \mathrm{Cl})$ & $P$ \\
\hline PICC & 43 & 61 & $9.692(6.414-14.646)$ & 0.000 & $4.268(2.501-7.282)$ & 0.000 \\
\hline Age (year, mean \pm standard deviation) & $61.27 \pm 12.63$ & $61.57 \pm 13.71$ & $0.998(0.989-1.007)$ & 0.724 & $1.005(0.995-1.015)$ & 0.325 \\
\hline Male & 156 & 1941 & $0.875(0.680-1.125)$ & 0.298 & $1.036(0.788-1.360)$ & 0.802 \\
\hline Malignant tumor & 48 & 79 & $8.493(5.787-12.464)$ & 0.000 & $1.856(1.038-3.321)$ & 0.037 \\
\hline Recent surgery & 80 & 160 & $7.593(5.857-10.797)$ & 0.000 & $4.056(2.503-6.571)$ & 0.000 \\
\hline Cerebral hemorrhage & 26 & 210 & $1.508(0.983-2.313)$ & 0.060 & $0.699(0.421-1.161)$ & 0.167 \\
\hline Ischemic stroke & 74 & 1539 & $0.403(0.306-0.531)$ & 0.000 & $0.562(0.411-0.768)$ & 0.000 \\
\hline Parkinson's disease & 8 & 222 & 0.407 (0.199-0.834) & 0.014 & $0.511(0.245-1.067)$ & 0.074 \\
\hline Infection & 26 & 311 & $0.984(0.646-1.499)$ & 0.939 & $1.597(1.016-2.508)$ & 0.042 \\
\hline $\mathrm{BMI} \geq 25$ & 29 & 209 & $1.712(1.136-2.579)$ & 0.010 & $1.325(0.838-2.096)$ & 0.229 \\
\hline Unconsciousness & 78 & 549 & $1.948(1.474-2.575)$ & 0.000 & $1.007(0.691-1.467)$ & 0.972 \\
\hline Critical illness & 57 & 117 & 7.010 (4.961-9.906) & 0.000 & $5.179(3.390-7.911)$ & 0.000 \\
\hline
\end{tabular}

Note: PICC refers to peripherally inserted central venous catheter; $B M I$ refers to body mass index; malignant tumor refers to cases treated within the prior six months; recent surgery refers to neurosurgery (over two hours) performed within six months; critical illness refers to the circumstance where patients have a high risk of disease variation or death

\section{D-dimer value before and after PICC insertion}

Ninety-two patients underwent D-dimer tests within seven days before and after catheterization. During the testing period, none of the patients were pregnant, took oral contraceptive, underwent major surgery, or had new tumor, heart failure, infection or trauma/fracture. The D-dimer value was significantly increased within seven days after the PICC insertion (Table $4, P<0.05$ ).

\section{Analysis of the predictive value of D-dimer for LEDVT}

ROC curves were plotted to evaluate the diagnostic sensitivity and specificity of D-dimer for LEDVT in patients with or without PICCs. The AUC of D-dimer in patients inserted with PICCs was 0.657 (Table 5; Fig. 1 and $95 \%$ CI: $0.549-0.765, P<0.05)$. The optimal critical value of D-dimer in patients inserted with PICCs was $0.675 \mathrm{mg} /$ $\mathrm{L}$, and the sensitivity, specificity, positive predictive value and negative predictive value of D-dimer were $48.28 \%$, $86.05 \%, 55.22$ and $82.35 \%$, respectively (Table 5).

The AUC of D-dimer in patients without PICC was 0.800 (Table 5; Fig. 2 and $95 \%$ CI: 0.769-0.830, $P<0.05$ ). The optimal critical value of D-dimer in patients without PICC was $0.665 \mathrm{mg} / \mathrm{L}$, and the sensitivity, specificity, positive predictive value and negative predictive value of

Table 3 Logistic regression analysis of D-dimer-related factors

\begin{tabular}{|c|c|c|c|c|c|c|}
\hline \multirow[t]{2}{*}{ Factors } & \multirow{2}{*}{$\begin{array}{l}\text { D-dimer } \\
\text { (>0.5 mg/L) } \\
n=597\end{array}$} & \multirow{2}{*}{$\begin{array}{l}\text { D-dimer } \\
(\leq 0.5 \mathrm{mg} / \mathrm{L}) \\
s=763\end{array}$} & \multicolumn{2}{|l|}{ Univariate } & \multicolumn{2}{|l|}{ Multivariate } \\
\hline & & & OR $(95 \% \mathrm{Cl})$ & $P$ & OR $(95 \% \mathrm{Cl})$ & $P$ \\
\hline $\mathrm{PICC}$ & 80 & 21 & $5.467(3.338-8.956)$ & 0.000 & $3.354(1.733-6.346)$ & 0.000 \\
\hline Age (year, mean \pm standard deviation) & $61.91 \pm 15.50$ & $60.17 \pm 14.46$ & $1.008(1.001-1.015)$ & 0.033 & $1.014(1.006-1.022)$ & 0.001 \\
\hline Male & 339 & 486 & $0.749(0.602-0.932)$ & 0.010 & $0.852(0.670-1.082)$ & 0.189 \\
\hline Malignant tumor & 85 & 38 & $3.167(2.126-4.720)$ & 0.000 & $0.785(0.430-1.435)$ & 0.432 \\
\hline Recent surgery & 158 & 64 & $3.931(2.872-5.381)$ & 0.000 & $4.944(3.015-8.107)$ & 0.000 \\
\hline Cerebral hemorrhage & 58 & 41 & $1.895(1.251-2.870)$ & 0.003 & $1.245(0.772-2.009)$ & 0.369 \\
\hline Ischemic stroke & 192 & 330 & $0.622(0.497-0.778)$ & 0.000 & $0.691(0.531-0.901)$ & 0.006 \\
\hline Parkinson's disease & 19 & 64 & $0.359(0.213-0.606)$ & 0.000 & $0.461(0.266-0.800)$ & 0.006 \\
\hline Infection & 121 & 85 & $2.028(1.500-2.740)$ & 0.000 & $3.078(2.218-4.271)$ & 0.000 \\
\hline $\mathrm{BMI} \geq 25$ & 42 & 57 & $0.937(0.620-1.148)$ & 0.759 & $0.641(0.400-1.026)$ & 0.064 \\
\hline Unconsciousness & 126 & 127 & $1.340(1.019-1.762)$ & 0.036 & $0.828(0.579-1.184)$ & 0.301 \\
\hline Critical illness & 78 & 38 & $2.867(1.915-4.294)$ & 0.000 & $2.844(1.754-4.613)$ & 0.000 \\
\hline
\end{tabular}

Note: PICC refers to peripherally inserted central venous catheter; $B M I$ refers to body mass index; malignant tumor refers to cases treated within the prior six months; recent surgery refers to neurosurgery (over two hours) performed within six months; critical illness refers to the circumstance where patients have a high risk of disease variation or death 
Table 4 D-dimer changes before and after PICC insertion

\begin{tabular}{llcll}
\hline & N & D-dimer $(\mathbf{m g} / \mathbf{L})$ & $\boldsymbol{t}$ & $\boldsymbol{P}$ \\
\hline Before PICC insertion & 92 & $0.86 \pm 0.84$ & -7.07 & 0.000 \\
After PICC insertion & 92 & $1.78 \pm 1.45$ & & \\
\hline
\end{tabular}

Note: PICC refers to peripherally inserted central venous catheter

D-dimer were $72.01 \%, 75.89 \%, 36.82$ and $93.25 \%$, respectively (Table 5).

The results show that the sensitivity, specificity, and positive predictive value of $\mathrm{D}$-dimer are not high. Generally, the negative predictive value of $\mathrm{D}$-dimer is used to exclude thrombosis. The negative predictive value of D-dimer in patients inserted with PICCs $(82.35 \%)$ is lower than in patients without PICC, suggesting D-dimer as an inappropriate predictor for thrombosis in patients inserted with PICCs.

\section{Discussion}

Venous thrombosis is a common vascular disorder in the general population. The annual incidence of venous thromboembolism in people of European descent is estimated to be 104 to 183 per 100,000 people [29]. DVT more often happens in lower extremities than in upper extremities. In USA, LEDVT has an annual incidence in about 187,000 individuals and is considered as the third common vascular disease following myocardial infarction and stroke [30]. Registro Informatizado de Enfermedad Trombo Embólica (RIETE) enrolled 37,366 patients with DVT, among which 35,094 (93.9\%) cases were LEDVT and the other $2272(6.1 \%)$ were UEDVT [31]. A recent retrospective cohort study of 83 patients hospitalized for DVT showed that 72 cases were LEDVT and 11 cases were UEDVT [32]. Foreign bodies in the vascular system are the most important independent risk factors of DVT [33-35]. A study of the general population showed that LEDVT occurred in 372 of 3790 patients inserted with PICCs during hospitalization [3]. In this study, 43 of the 270 LEDVT patients were inserted with PICCs. Consistently, we found that PICC insertion increased the risk of LEDVT by 4 times after risk adjustment. LEDVT occurred in 43 of 104 patients inserted with PICCs 6.8 days (an average value) after catheterization. The median time from index to LEDVT was five days, which is similar to the time for UEDVT. PICC-related UEDVT occurs within 12 days after catheterization, and the median time for UEDVT is 8 days [36]. In addition to the PICC insertion, factors including malignancy, recent surgery, infection, BMI $\geq$ 25 and unconsciousness were also associated with the increased risk of LEDVT.

The concentration of D-dimer is considered a gold biochemical standard for assessing both coagulation activation and fibrin digestion [37] and for ruling out venous thrombosis in patients with low-median clinical probability [38-40]. Other conditions such as infection, cancer, chronic inflammation, aging, pregnancy, recent surgery, and trauma can also increase the concentration of D-dimer by accelerating fibrin production or breakdown [41]. A retrospective study of 1647 patients showed that the most common cause of positive D-dimer was infection, followed by venous thrombosis, syncope, heart failure, trauma, and cancer [42]. After excluding these factors, we investigated the D-dimer concentration within seven days before PICC catheterization. Evidence showed that the risk of PICC line-related DVT was increased in the first two weeks after PICC insertion [15]. We found that the concentration of D-dimer within seven days after PICC insertion was higher than that before PICC insertion, indicating a PICC-induced increase in D-dimer. Moreover, recent surgery, infection, and cerebral hemorrhage were also associated with the increase in $\mathrm{D}$-dimer. $\mathrm{D}$-dimer cannot be used to exclude venous thrombosis in patients inserted with PICCs.

Thrombosis is a natural process activated by internal and external pathways which stimulate series of coagulation in the body and eventually lead to fibrin rich thrombus. Endothelial injury is a coagulation inciting event. Fibrinolysis and destruction of the blood clot are induced following coagulation to maintain homeostasis. DVT occurs when the coagulation process is not restrained or the mechanism of decomposing blood clots is overloaded [43]. A PICC occupies nearly half of the inner diameter of a vein, which causes a slowing of the local blood flow. The stasis of blood flow can induce micro venous thrombosis and activate the coagulation system in the process of reflux, leading to a larger range of DVT [43]. We suspect that PICC insertion is a risk factor of LEDVT and results in a general increase in Ddimer concentration. By the joint action of other risk factors, DVT can spread beyond the PICC vascular bed.

Table 5 Analysis of the predictive value of D-dimer for LEDVT

\begin{tabular}{|c|c|c|c|c|c|c|c|}
\hline & $\begin{array}{l}\text { Optimal } \\
\text { critical value }\end{array}$ & Sensitivity & Specificity & $\begin{array}{l}\text { Positive } \\
\text { predictive } \\
\text { value }\end{array}$ & $\begin{array}{l}\text { Negative } \\
\text { predictive } \\
\text { value }\end{array}$ & $\begin{array}{l}\text { Area under } \\
\text { ROC curve }\end{array}$ & $\begin{array}{l}95 \% \text { confidence } \\
\text { interval }\end{array}$ \\
\hline $\begin{array}{l}\text { The level of D-dimer in } \\
\text { patients with PICC }\end{array}$ & 0.675 & $48.28 \%$ & $86.05 \%$ & $55.22 \%$ & $82.35 \%$ & 0.657 & $0.549-0.765$ \\
\hline $\begin{array}{l}\text { The level of D-dimer in } \\
\text { patients without PICC }\end{array}$ & 0.665 & $72.01 \%$ & $75.89 \%$ & $36.82 \%$ & $93.25 \%$ & 0.800 & $0.769-0.830$ \\
\hline
\end{tabular}




\section{ROC curve}

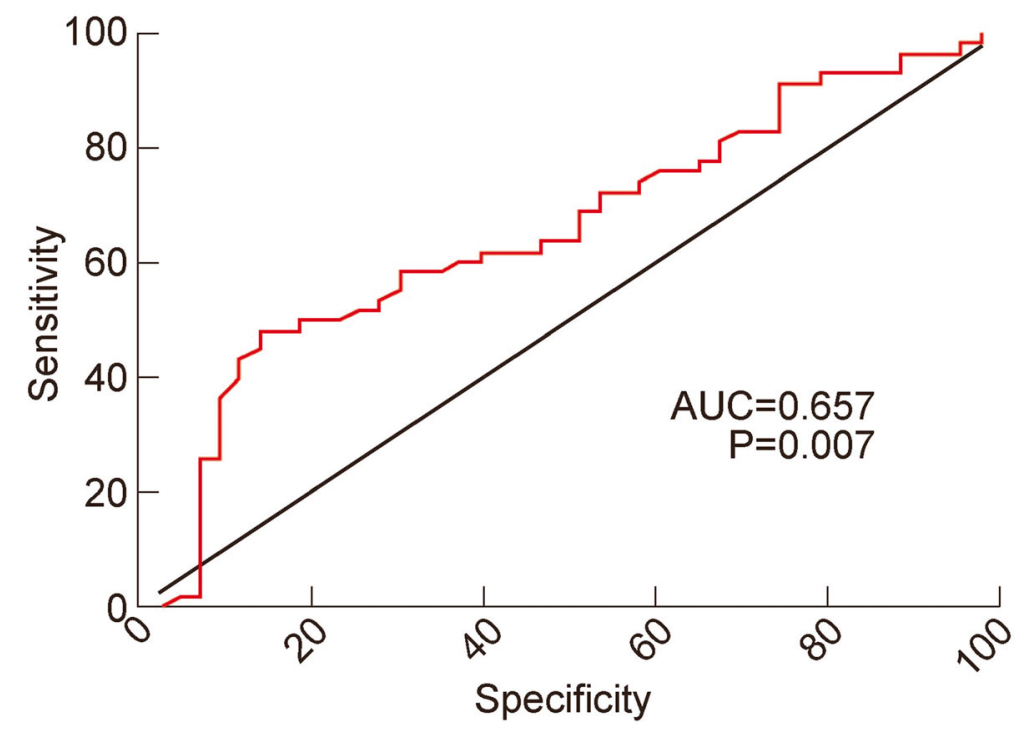

Fig. 1 Area under ROC curve of D-dimer for diagnosis of LEDVT in patients inserted with PICCS. PICC, peripherally inserted central venous catheter; LEDVT, lower extremity deep venous thrombosis; ROC, receiver operating characteristic curve

This study provides novel insights for the clinical evaluation of LEDVT. A previous systematic search of the literature for PICC-related DVT summarized the risk factors, symptoms, diagnosis, management and prevention of this event [44]. However, how PICC insertion is related to DVT is barely known. In this study, the concentration of D-dimer was first used to explain the possible relationship between PICC inserted in upper extremities and LEDVT. A PICC inserted in upper extremities increases the risk of LEDVT, which inspires clinicians to consider the complications of PICC insertion. For patients with a high risk of thrombosis, it is necessary for a specialized nursing team to weigh the side-effect and benefit of PICC or to consider an alternative vascular access. Moreover, clinicians are suggested to use a prophylactic anticoagulant regimen in patients

\section{ROC curve}

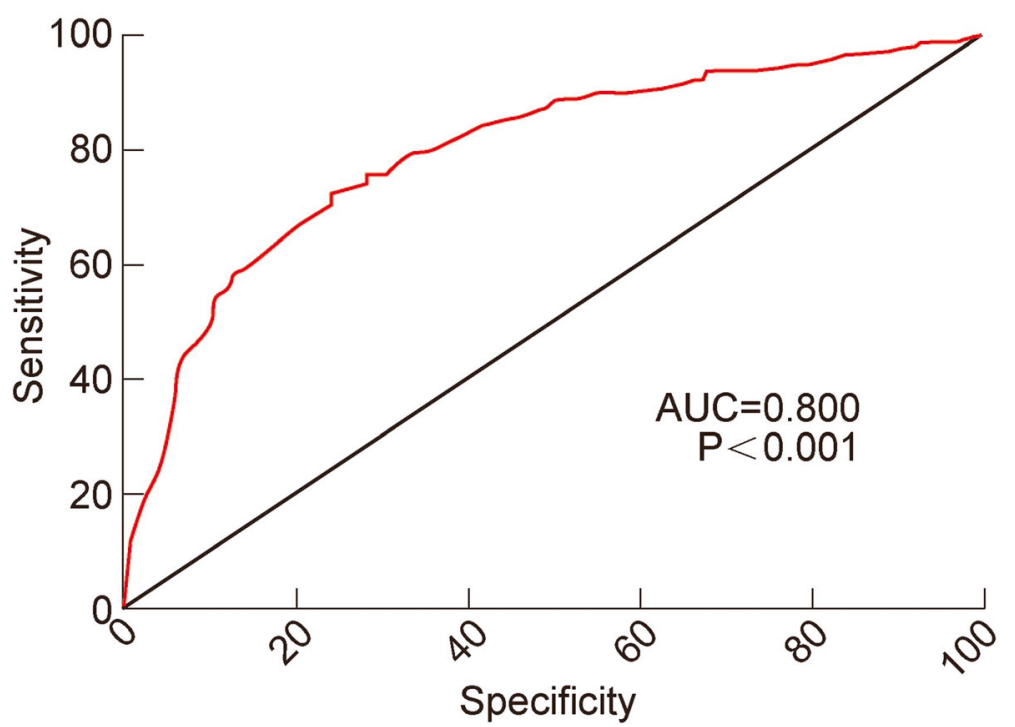

Fig. 2 Area under ROC curve of D-dimer for diagnosis of LEDVT in patients without PICC. PICC, peripherally inserted central venous catheter; LEDVT, lower extremity deep venous thrombosis; ROC, receiver operating characteristic curve 
at high risk of thrombosis who need PICC insertion. This study suggested a connection between PICC insertion and subsequent LEDVT and another possibility is that the PICC insertion may be a marker of a sicker and higher risk patient. In this regards, we have added the factor critical illness and main risk factors for LEDVT for logistic regression analysis and the results supported this possibility.

Despite the significance, limitations exist in this study. Firstly, the data of asymptomatic UEDVT was not collected to determine whether PICC insertion conferred a greater risk of DVT in upper extremities than in lower extremities. Secondly, this study was limited to the neurology department.

\section{Conclusions}

PICC insertion increases the value of D-dimer and is an important risk factor of LEDVT. The predictive value of D-dimer in patients inserted with PICCs is lower than in patients without PICC. D-dimer is unsuitable for routine examination to exclude LEDVT in patients inserted with PICCs.

\section{Abbreviations}

PICC: Peripherally Inserted Central venous Catheter; LEDVT: Lower extremity deep venous thrombosis; UEDVT: Upper extremity deep venous thrombosis

\section{Acknowledgements}

Not applicable.

\begin{abstract}
Authors' contributions
Wanli Liu participated in data acquisition and management of the study, analyzed and interpreted the data, and drafted and revised the manuscript. Lianxiang He and Wenjing Zeng and Liqing Yue participated in data interpretation, and revision of the manuscript. Jie Wei and Shuangshuang Zeng participated in protocol drafting and study management. Zhicheng Gong was the trial manager who designed, initiated and managed the study, participated in data acquisition and interpretation, and revised the manuscript. All authors read and approved the final manuscript.
\end{abstract}

\section{Funding}

This research was supported by the grants from the Natural Science Foundation of Hunan Province (2020JJ5880) and the Finance Department Project of Hunan Province, People's Republic of China (No. [2019] 60) and Hunan Science and Technology Innovation Project (2018SK52606).

\section{Availability of data and materials}

The datasets used and/or analyzed during the current study are available from the corresponding author on reasonable request.

\section{Declarations}

Ethics approval and consent to participate

The study was approved by the ethics committee of Xiangya Hospital of Central South University $(202,004,327)$ and was conducted according to the Helsinki Declaration of Ethical Principles for Medical Research involving Human Subjects.

\section{Consent for publication}

Not applicable.

\section{Competing interests}

The authors declare that they have no competing interests.

\section{Author details}

${ }^{1}$ Teaching and Research Section of Clinical Nursing, Xiangya Hospital, Central South University, Changsha, Hunan, People's Republic of China. ${ }^{2}$ Institute for Rational and Safe Medication Practices, National Clinical Research Center for Geriatric Disorders, Xiangya Hospital, Central South University, Changsha, Hunan, People's Republic of China. ${ }^{3}$ Department of Pharmacy, Xiangya Hospital, National Clinical Research Center for Geriatric Disorders, Central South University, Hunan 410008 Changsha, People's Republic of China.

Received: 31 August 2020 Accepted: 22 March 2021

Published online: 09 April 2021

\section{References}

1. Commission NHAF. Technical operation specifications for intravenous therapy nursing. China nursing management. 2014;1 (14):1-4,

2. Song $L$, et al. Malposition of peripherally inserted central catheter: experience from 3012 cancer patients. Int J Nurs Pract. 2014;20(4): 446-9.

3. Chopra V. G.M.B.S., The Association between Upper and Lower Extremity Deep Vein Thrombosis and Peripherally Inserted Central Catheters: Think Below the Waist Annual Scientific Conference Abstract. Society of Hospital Medicine, 2014(Las Vegas, NV.).

4. Olaf M, et al. Deep Venous Thrombosis. Emerg Med Clin North Am. 2017; 35(4):743-70.

5. O'Brien J, et al. Insertion of PICCS with minimum number of lumens reduces complications and costs. J Am Coll Radiol. 2013;10:864-8.

6. Aw A, et al. Incidence and predictive factors of symptomatic thrombosis related to peripherally inserted central catheters in chemotherapy patients. Thromb Res. 2012;130(3):323-6.

7. Chemaly RF, et al. Venous thrombosis associated with peripherally inserted central catheters: a retrospective analysis of the Cleveland Clinic experience. Clin Infect Dis. 2002;34(9):1179-83.

8. Cortelezzia A, et al. Central venous catheter-related complications in patients with hematological malignancies: a retrospective analysis of risk factors and prophylactic measures. Leuk Lymphoma. 2003;44(9):1495-501.

9. King MM, et al. Peripherally inserted central venous catheter-associated thrombosis: retrospective analysis of clinical risk factors in adult patients. South Med J. 2006;99(10):1073-7.

10. Paauw JD, et al. The incidence of PICC line-associated thrombosis with and without the use of prophylactic anticoagulants. JPEN J Parenter Enteral Nutr. 2008:32(4):443-7.

11. Itkin $\mathrm{M}$, et al. Peripherally inserted central catheter thrombosis - reverse tapered versus nontapered catheters: a randomized controlled study. J Vasc Interv Radiol. 2014;25(1):85-91.

12. Liem TK, et al. Peripherally inserted central catheter usage patterns and associated symptomatic upper extremity venous thrombosis. J Vasc Surg. 2012:55(3):761-7.

13. Olson JD. D-dimer: An Overview of Hemostasis and Fibrinolysis, Assays, and Clinical Applications. Adv Clin Chem. 2015;69(2):1-46.

14. Easaw JC, et al. Canadian consensus recommendations on the management of venous thromboembolism in patients with cancer. Part 2: treatment. Curr Oncol. 2015;22(2):144-55.

15. Greene MT, et al. The Association Between PICC Use and Venous Thromboembolism in Upper and Lower Extremities. The American Journal of Medicine. 2015;128(9):986-93.e1.

16. Al-Asadi $\mathrm{O}$, Almusarhed $\mathrm{M}$, Eldeeb $\mathrm{H}$. Predictive risk factors of venous thromboembolism (VTE) associated with peripherally inserted central catheters (PICC) in ambulant solid cancer patients: retrospective single Centre cohort study. Thrombosis Journal. 2019;17(2):1-7.

17. van der Straaten Mark Roest Fred Haas Philip. De Groot Rob G, Fijnheer FHJH. Elevated levels of D-dimer and fragment $1+2$ upon central venous catheter insertion and factor $\mathrm{V}$ Leiden predict subclavian vein thrombosis. the hematology journal, 2005. 4(90): 499-504.

18. Chen Q, Z.Z.D.H. and Zhi ZFAZ. Perioperative Venous Thromboembolism (VTE) Prophylaxis in Thoracic Cancer Patients: Chinese Experts Consensus Interpretation of Clinical Significance of D-dimer. Zhongguo Fei Ai Za Zhi. 2019:12(22):761-6.

19. Kanno Y, et al. Elevation of plasma D-dimer is closely associated with venous thrombosis produced by double-lumen catheter in pre-dialysis patients. Nephrology Dialysis Transplantation. 2007;22(4):1224-7. 
20. Zhang D, et al. Diagnostic accuracy of biomarker D-dimer in patients after stroke suspected from venous thromboembolism: A diagnostic metaanalysis. Clin Biochem. 2019;63(2):126-34.

21. Jeffrey I, Weitz MABC. A Test in Context: D-Dimer. JOURNAL OF THE AMERIC AN COLLEGE OF CARDIOLOGY, 2017. 70(19): p. 2411-2419.

22. Lim W, et al. American Society of Hematology 2018 guidelines for management of venous thromboembolism: diagnosis of venous thromboembolism. Blood Adv. 2018;2(22):3226-56.

23. Chopra $V$, et al. Risk of venous thromboembolism associated with peripherally inserted central catheters: a systematic review and metaanalysis. Lancet. 2013;382(9889):311-25.

24. CLSI. Quantitative. D-dimer for exclusion of venous thromboembolic disease. CLSI. document H59-A Wayne. PA: Clinical and Laboratory Standard Institute; 2011

25. Di Nisio M, Squizzato A, Rutjes AW, et al. Diagnostic accuracy of D-dimer test for exclusion of venous thromboembolism: a systematic review. J Thromb Haemost. 2007;5:296-304.

26. Stein PD, Beemath A, Meyers FA, Skaf E, Sanchez J, Olson RE. Incidence of venous thromboembolism in patients hospitalized with cancer. Am J Med. 2006;119(1):60-8.

27. Menéndez JJ, Verdú C, Calderón B, et al. Incidence and risk factors of superficial and deep vein thrombosis associated. with peripherally inserted central catheters in children. J Thromb Haemost.2016;14(11):2158-68.

28. Kearon C, Akl EA, Ornelas J, et al. Antithrombotic Therapy for VTE disease: CHEST Guideline and Expert Panel Report. Chest. 2016;149(2):315-52.

29. Heit JA. Epidemiology of venous thromboembolism. Nat Rev Cardiol. 2015; 12(8):464-74.

30. Heit JA. The epidemiology of venous thromboembolism in the community: implications for prevention and management. J Thromb Thrombolysis. 2006;21(1):23-9.

31. Cote LP, et al. Comparisons Between Upper and Lower Extremity Deep Vein Thrombosis: A Review of the RIETE Registry. Clin Appl Thromb Hemost. 2017:23(7):748-54.

32. Rokosh RS, et al. High Prevalence and Mortality Associated with Upper Extremity Deep Venous Thrombosis in Hospitalized Patients at a Tertiary Care Center. Ann Vasc Surg. 2020;65:55-65.

33. Frolova $\mathrm{Al}$, et al. Complications of peripherally inserted central catheters in pregnancy: a systematic review and meta-analysis. J Matern Fetal Neonatal Med. 2020. https://doi.org/10.1080/14767058.2020.1769591.

34. Jiang $M$, et al. Risk of venous thromboembolism associated with totally implantable venous access ports in cancer patients: a systematic review and meta-analysis. J Thromb Haemost. 2020. https://doi.org/10.1111/jth.14930.

35. Heil J, et al. Deep Vein Thrombosis of the Upper Extremity. Dtsch Arztebl Int. 2017:114(14):244-9.

36. Fletcher JJ, et al. The clinical significance of peripherally inserted central venous catheter-related deep vein thrombosis. Neurocrit Care. 2011;15(3):454-60.

37. Gaffney PJ, Lane DA, Kakkar W, et al. Characterisation of a soluble D dimerE complex in crosslinked fibrin digests. Thromb Res. 1975;7:89-99.

38. Thachil J, Lippi G, Favaloro EJ. D-dimer testing: laboratory aspects and current issues. Methods Mol Biol. 2017;1646:91-104.

39. Lippi G, Cervellin G, Casagranda I, et al. D-dimer testing for suspected venous thromboembolism in the emergency department. Consensus document of AcEMC, CISMEL, SIBioC, and SIMeL. Clin Chem Lab Med. 2014;52:621-8.

40. Kamolratanapiboon K. Chaicharoen Tantanate. (2019) Inappropriate use of D-dimer and impact on the test characteristics for deep vein thrombosis exclusion. Scandinavian Journal of Clinical and Laboratory Investigation. 2019;79(6):431-436

41. Linkins LA, Takach Lapner S. Review of D-dimer testing: good, bad, and ugly. Int J Lab Hematol. 2017;39(Suppl 1):98-103

42. Lippi G, Bonfanti L, Saccenti C, et al. Causes of elevated D-dimer in patients admitted to a large urban emergency department. Eur J Intern Med. 2014; 25:45-8.

43. Hattab Y, et al. Deep Venous Thrombosis of the Upper and Lower Extremity. Crit Care Nurs Q. 2017;40(3):230-6.

44. Fallouh N, McGuirk HM, Flanders SA, Chopra V. Peripherally Inserted Central Catheter-associated Deep Vein Thrombosis: A Narrative Review. Am J Med. 2015;128(7):722-38.

\section{Publisher's Note}

Springer Nature remains neutral with regard to jurisdictional claims in published maps and institutional affiliations.

\section{Ready to submit your research? Choose BMC and benefit from:}

- fast, convenient online submission

- thorough peer review by experienced researchers in your field

- rapid publication on acceptance

- support for research data, including large and complex data types

- gold Open Access which fosters wider collaboration and increased citations

- maximum visibility for your research: over $100 \mathrm{M}$ website views per year

At $\mathrm{BMC}$, research is always in progress.

Learn more biomedcentral.com/submissions 